\title{
Dysregulated brain creatine kinase is associated with hearing impairment in mouse models of Huntington disease
}

\author{
Yow-Sien Lin, ${ }^{1,2,3}$ Chiung-Mei Chen, ${ }^{4}$ Bing-wen Soong, ${ }^{5}$ Yih-Ru Wu, ${ }^{4}$ Hui-Mei Chen, ${ }^{3}$ \\ Wen-Ying Yeh, 6 Dai-Rong Wu,6 Yi-Jun Lin, ${ }^{6}$ Paul Wai-Fung Poon,7 \\ Mei-Ling Cheng, ${ }^{8}$ Chih-Hung Wang, ${ }^{6,9}$ and Yijuang Chern ${ }^{1,2,3}$

\begin{abstract}
${ }^{1}$ Molecular Medicine Program and 2Institute of Neuroscience, National Yang-Ming University, Taipei, Taiwan. ${ }^{3}$ Division of Neuroscience, Institute of Biomedical Sciences, Academia Sinica, Taipei, Taiwan. ${ }^{4}$ Department of Neurology, Chang Gung Memorial Hospital, Linkou Medical Center and College of Medicine, Chang-Gung University, Taoyuan, Taiwan. ${ }^{5}$ Department of Neurology, Yang-Ming University School of Medicine and Neurological Institute, Taipei Veterans General Hospital, Taipei, Taiwan. ${ }^{6}$ Department of Otolaryngology — Head and Neck Surgery, Tri-Service General Hospital, Taipei, Taiwan. ${ }^{7}$ Institute of Physiology, National Cheng Kung University, Tainan, Taiwan. ${ }^{8}$ Graduate Institute of Medical Biotechnology, Chang Gung University, Kweisan, Taoyuan, Taiwan. Institute of Microbiology and Immunology, National Defense Medical Center, Taipei, Taiwan.
\end{abstract}

\begin{abstract}
Huntington disease (HD) is a degenerative disorder caused by expanded CAG repeats in exon 1 of the huntingtin gene $(H T T)$. Patients with late-stage HD are known to have abnormal auditory processing, but the peripheral auditory functions of HD patients have yet to be thoroughly assessed. In this study, $19 \mathrm{HD}$ patients (aged 40-59 years) were assessed for hearing impairment using pure-tone audiometry and assessment of auditory brainstem responses (ABRs). PTA thresholds were markedly elevated in HD patients. Consistent with this, elevated ABR thresholds were also detected in two mouse models of HD. Hearing loss thus appears to be an authentic symptom of HD. Immunohistochemical analyses demonstrated the presence of mutant huntingtin that formed intranuclear inclusions in the organ of Corti of HD mice, which might interfere with normal auditory function. Quantitative RT-PCR and Western blot analyses further revealed reduced expression of brain creatine kinase (CKB), a major enzyme responsible for ATP regeneration via the phosphocreatine-creatine kinase (PCr-CK) system, in the cochlea of HD mice. Treatment with creatine supplements ameliorated the hearing impairment of HD mice, suggesting that the impaired PCr-CK system in the cochlea of HD mice may contribute to their hearing impairment. These data also suggest that creatine may be useful for treating the hearing abnormalities of patients with HD.
\end{abstract}

\section{Introduction}

Huntington disease (HD) is a dominant neurodegenerative disorder that usually becomes established in middle age. The clinical features include uncontrollable chorea movement, cognitive impairment, and psychiatric syndromes (1). The number of CAG repeats in exon 1 of the huntingtin gene (HTT) of HD patients is known to exceed 35 . The expanded CAG repeat is translated into a polyglutamine (polyQ) stretch at the $\mathrm{N}$-terminus of the Htt protein. PolyQ-expanded mutant Htt forms nuclear and neutrophil aggregates and preferentially affects the striatum and the cortex in the brain and several peripheral tissues $(2,3)$. Recent studies demonstrate that late-stage HD patients have abnormal auditory sensory memory and that auditory processing is altered in $\operatorname{HD}$ patients $(4,5)$. Nevertheless, the peripheral auditory functions of HD patients have not yet been thoroughly assessed.

Brain-type creatine kinase $(\mathrm{CKB})$ is a cytosolic enzyme that regulates ATP regeneration and energy homeostasis. It controls ATP concentration through the transfer of high-energy phosphate from phosphocreatine (PCr) to ADP. Tissues that consume large amounts of energy (including the brain and the cardiac muscles)

Authorship note: Yow-Sien Lin, Chiung-Mei Chen, and Bing-wen Soong contributed equally to this work.

Conflict of interest: The authors have declared that no conflict of interest exists. Citation for this article: J Clin Invest. 2011;121(4):1519-1523. doi:10.1172/JCI43220. express high levels of creatine kinases (CKs), implying a critical role of CKs in maintaining appropriate ATP concentrations (6). In the inner ear, stereocilia of hair cells mediate mechanosensing, a very energy-demanding process. Because stereocilia contain no mitochondria, the PCr-CK system thus plays a critical role in maintaining their supply. The importance of the PCr-CK system has been demonstrated using a CKB-knockout mouse model that preferentially exhibits high-tone hearing loss (7). In the postmortem brains of HD patients, CKB is oxidatively modified and progressively inhibited as the disease advances (8). We thus hypothesized that $\mathrm{CKB}$ in the HD cochlea might also be dysregulated, as was shown in the brain (8), and subsequently causes hearing impairment.

\section{Results}

We recruited 19 middle-aged HD patients (aged 40-59 years; 11 men and 8 women, Supplemental Table 1; supplemental material available online with this article; doi:10.1172/JCI43220DS1) and 20 age-matched non-HD controls (10 men and 10 women) to determine their hearing thresholds using pure-tone audiometry. Patients had higher pure-tone thresholds (PTTs) compared with the non-HD controls (Figure 1A and Supplemental Table 2) or with a larger group of age-matched controls recruited in the National Health and Nutrition Examination Survey (NHANES) (ref. 9 and Supplemental Figure 1C). Individuals are characterized as being hearing impaired if the hearing level of the better-hearing ear has a mean 4-frequency pure-tone average (4F-PTA; 500, 

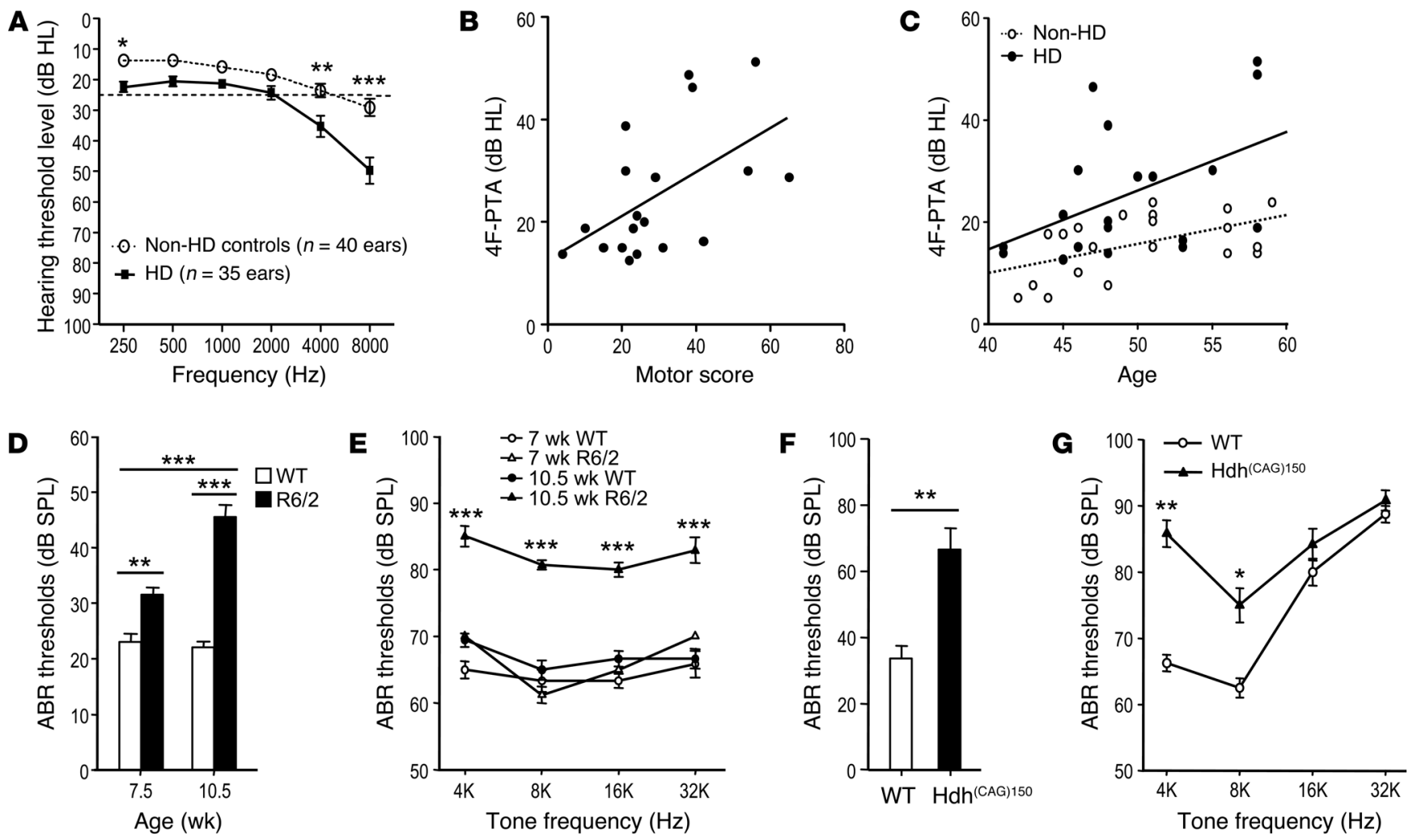

Figure 1

Hearing loss in HD. (A) Mean value of the PTTs of HD patients at each specific frequency ( $n=35$ ears) and of the non-HD controls ( $n=40$ ears). The dotted line indicates the normal hearing level $(25 \mathrm{~dB} \mathrm{HL}) .{ }^{*} P<0.05,{ }^{* \star} P<0.01,{ }^{\star \star \star} P<0.001$. Three ears of HD patients were excluded because of middle ear pathologies. (B) The 4F-PTAs of HD patients were significantly correlated with the total motors score of HD patients $(P<0.05, r=0.5274$; Pearson's correlation). (C) The 4F-PTAs of HD patients $(n=19, P<0.05, r=0.4789)$ and non-HD controls $(n=20$, $P<0.05, r=0.5196$ ) were associated with age. ABR thresholds of WT and R6/2 mice were measured by click (D) and tone-burst (E) stimuli at the ages of 7.5 and 10.5 weeks ( $n=10$ per group). Click ABR $(\mathbf{F})$ and tone-burst ABR $(\mathbf{G})$ analysis of WT $(n=4)$ and Hdh ${ }^{(C A G) 150}(n=6)$ mice at the age of 15 months. SPL, sound pressure level. ${ }^{\star} P<0.05,{ }^{* *} P<0.01,{ }^{* *} P<0.001$.

$1,000,2,000$, and $4,000 \mathrm{~Hz}$ ) of more than 25 decibels hearing level (dB HL) (10). The prevalence of hearing impairment in HD patients $(42.1 \%$, 95\% CI: $20.3 \%-66.5 \%)$ was also significantly higher than that in the non-HD controls $(0 \%, 95 \%$ CI: $0 \%-16.9 \%$, $P<0.0012)$ or a larger group of age-matched NHANES controls (ref. 9 and Supplemental Table 3). The severity of disease in HD patients was evaluated using the Unified Huntington's Disease Rating Scale (UHDRS), which rates motor function, independence, and functional capacity (11). Greater disease severity is associated with higher scores for motor function and lower scores for independence and functional capacity. The hearing deficit of HD patients was closely associated with the total motor score (Figure 1B), but not with the independence scale and functional capacity scores, of HD patients (Supplemental Figure 2, A and B). No correlation existed between hearing impairment and CAG repeat length or disease duration (Supplemental Figure 2, C and D). Collectively, the data indicate that hearing deficit may serve as an indicator of disease severity or progression of HD. The hearing deficit in both non-HD controls $(P=0.0189$ and $r=0.5196)$ and HD patients $(P=0.038$ and $r=0.4789)$ correlated well with age (Figure 1C). A multiple linear regression analysis showed a significant difference in the age dependence of hearing thresholds between HD patients and non-HD controls $(P<0.001$, Supplemental Table 4$)$.
Next, we examined whether the impaired hearing of HD patients resulted from retrocochlear lesion by determining the latencies of auditory brainstem response (ABR) waveforms in HD patients and non-HD controls. Analysis of the data revealed the absence of significant disease differences in latency and inter-peak intervals of ABR (Supplemental Figure 3, A-C). Moreover, there was no difference in ABR latency and inter-peak intervals between a transgenic HD mouse model (R6/2; ref. 12) and WT mice (Supplemental Figure 3). These results indicate that the central auditory transmission pathway is not affected in HD patients or mice. The impaired hearing sensitivity of HD patients therefore might be associated more with peripheral auditory defects and less with the deficits of the auditory brainstem pathway (i.e., retrocochlear lesion).

Using ABR threshold analyses, we assessed the hearing sensitivity of R6/2 mice and a knock-in HD mouse model (Hdh ${ }^{(\mathrm{CAG}) 150}$; ref. 13). R6/2 mice had higher ABR thresholds for both click and tone-burst stimuli starting from the age of 7.5 weeks, when motor deficit had not yet been observed (Figure 1D and Supplemental Figure 4B). As the disease progressed, the hearing impairment and motor deficit became significant at the age of 10.5 weeks (Figure 1, D and E, and Supplemental Figure 5). Similar hearing loss was observed in knockin $\mathrm{Hdh}(\mathrm{CAG}) 150$ mice. These mice show neurological abnormalities at approximately $10-14$ months of age (13). We thus recorded ABR 
A

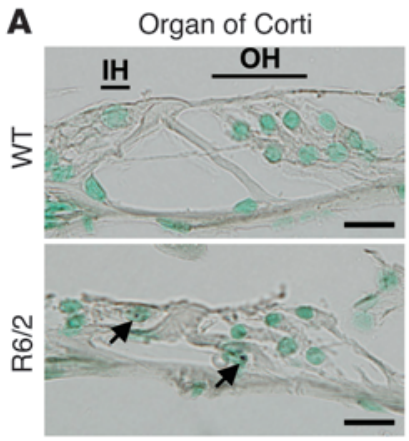

C

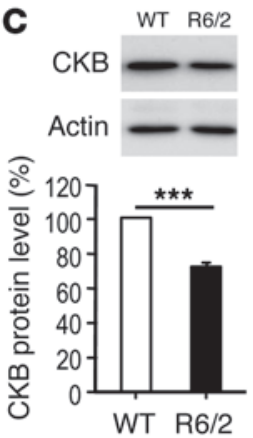

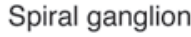

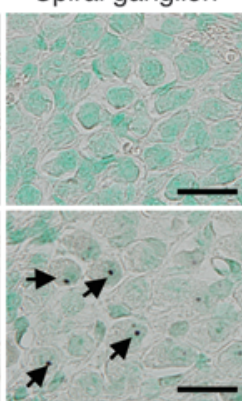

D E

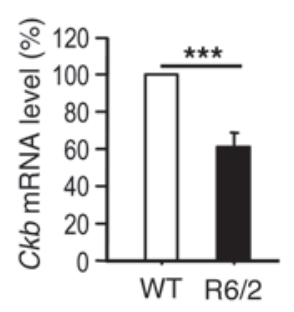

5
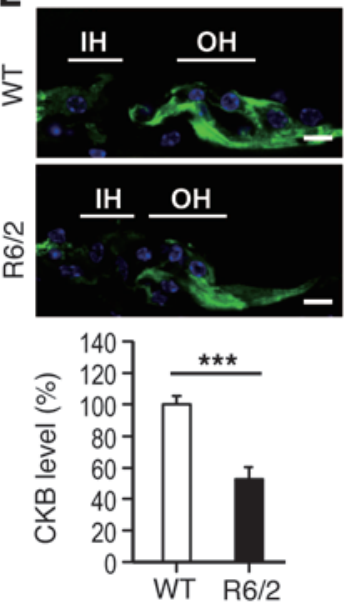

B

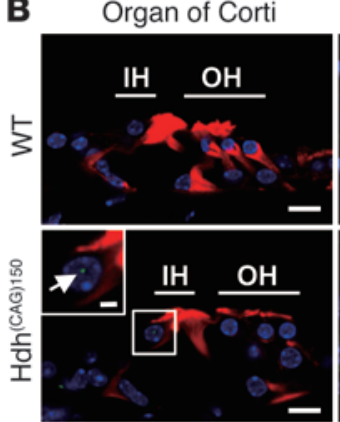

Spiral ganglion

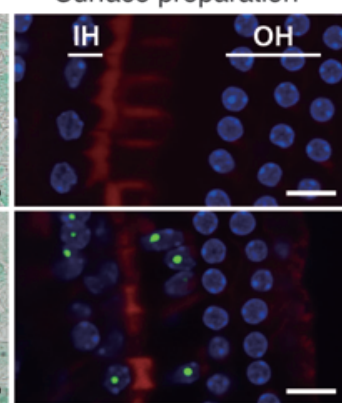

$\mathbf{F}$

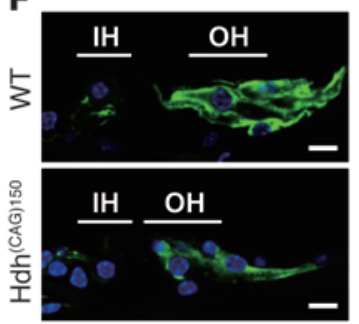

G
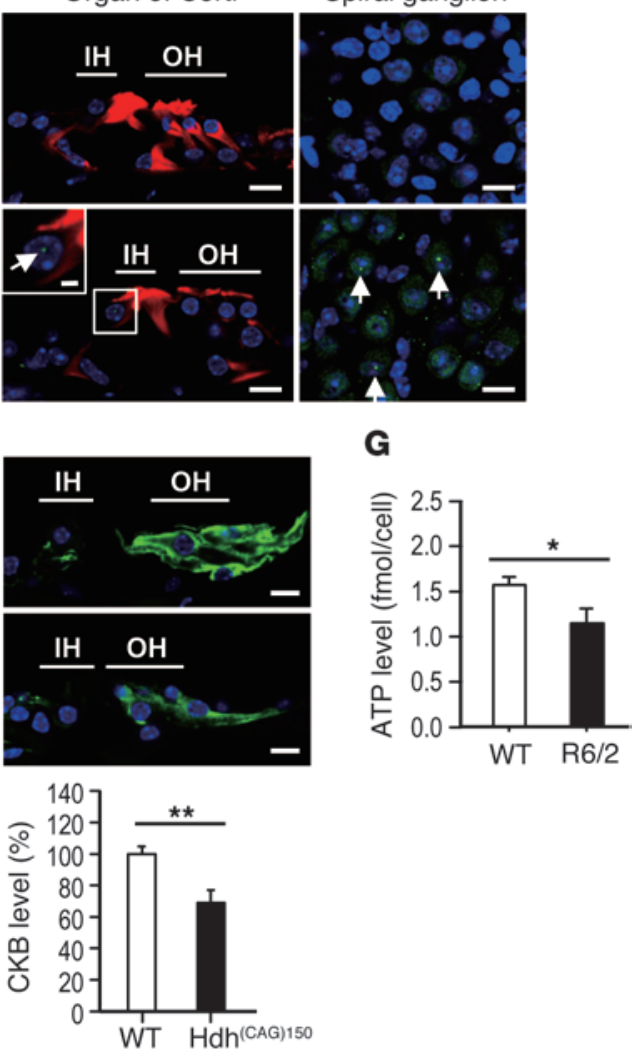

Figure 2

Auditory dysfunction in HD mice. (A) Cochleae (mid-basal turn) of 10.5-week-old mice were visualized by immunohistochemistry (Htt, dark brown deposits; nuclei, green). NIls (arrows) were detected in the spiral ganglion (middle panels) and in the organ of Corti (left panels) of R6/2 mice. Surface preparations (right panels; mid-basal turn) of 10.5-week-old mice were performed to detect NIls using an anti-Htt antibody (green), nuclei using Hoechst 33258 (blue), and the organ of Corti using rhodamine phalloidin (red). IH, inner hair cell; OH, outer hair cell. (B) Cryosections of the cochlea of 15-month-old mice (mid-basal turn) were stained with an anti-ubiquitin antibody (green) and with rhodamine phalloidin (red). Arrows indicate NIls. (C) Total protein (30 $\mu \mathrm{g}$ per lane) from the cochleae of 10.5-week-old WT and R6/2 mice was analyzed for the expression of CKB protein, which was normalized to that of actin. (D) RNA from cochleae of 10.5-week-old WT and R6/2 mice ( $n=6$ per group) was used to determine the level of the $C k b$ transcript using an RT-qPCR method. The expression levels of $C k b$ were normalized to those of a reference gene (Gapdh). (E) CKB immunostaining (green) of the cochlea of 12-week-old WT and R6/2 mice. (F) CKB immunostaining (green) of the cochleae of

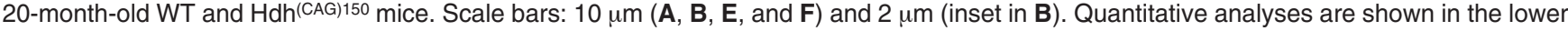
panels (C, E, and F). (G) ATP level in isolated hair cells of 12 -week-old WT and R6/2 mice. ${ }^{*} P<0.05,{ }^{* *} P<0.01$, and ${ }^{* * *} P<0.001$.

from $\mathrm{Hdh}^{(\mathrm{CAG}) 150}$ mice at the age of 15 months. Thresholds measured by click ABR analysis were significantly higher in $\mathrm{Hdh}^{(\mathrm{CAG}) 150}$ mice than in WT mice (Figure 1F). ABR thresholds for tone bursts of 4, 8, 16 , and $32 \mathrm{kHz}$ of $\mathrm{Hdh}^{(\mathrm{CAG}) 150}$ mice also revealed a trend toward deteriorated hearing ability and a significant elevation of the threshold levels at 4 and $8 \mathrm{kHz}(P<0.01$ and $P<0.05$, respectively). Because of the late onset of symptoms in $\mathrm{Hdh}^{(\mathrm{CAG}) 150}$ mice, the WT controls (age matched at 15 months) had developed presbycusis at 16 and $32 \mathrm{kHz}$. Thus, the ABR thresholds of $\mathrm{Hdh}^{(\mathrm{CAG}) 150}$ and WT mice did not differ at 16 and $32 \mathrm{kHz}$ (Figure $1 \mathrm{G}$ ).

We next performed immunohistochemical staining of the cochlea and hair cells of R6/2 and $\mathrm{Hdh}^{(\mathrm{CAG}) 150}$ mice to assess whether mutant Htt forms neuronal intranuclear inclusions (NIIs). We detected NIIs in the spiral ganglion and the organ of Corti of R6/2 mice, but not in WT mice (Figure 2A). Because the anti-Htt antibody (mAb5374, Chemicon) reacts poorly with mouse Htt, we used an anti-ubiquitin antibody to detect NIIs in $\mathrm{Hdh}^{(\mathrm{CAG}) 150}$ mice as described previously (13). Similar to R6/2 mice, the $\mathrm{Hdh}^{(\mathrm{CAG}) 150}$ mice also contained NIIs in the spiral ganglion and organ of Corti (Figure 2B), demonstrating the existence of NIIs in the cochlea of HD mice. NIIs were present mostly in the organ of Corti within the basal, middle-basal, and apical turns of the cochlear duct.

Because oxidation of CKB and the subsequent suppression of $\mathrm{CKB}$ activity have been reported in the brains of HD mice and patients $(8,14)$ and because CKB is critical for maintaining hearing function (7), we hypothesized that CKB might be dysregulated in the cochlea of HD mice with hearing impairment. Western blot, quantitative RT-PCR, and immunohistochemical analyses revealed that the protein and transcript levels of CKB were lower in the R6/2 compared with WT mouse cochlea (Figure 2, C-E). Similar reduction in CKB expression was also found in the cochlea of $\mathrm{Hdh}^{(\mathrm{CAG}) 150}$ mice (Figure $2 \mathrm{~F}$ ). Our data suggest that the energy deficiency caused by $\mathrm{CKB}$ reduction mediates the hearing deficit of HD mice. Consistent with this hypothesis, the ATP level of isolated hair cells from the R6/2 cochlea was lower than that detected in WT mice (Figure $2 \mathrm{G}$ ). 


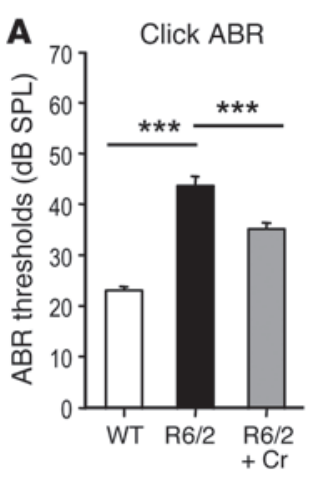

D

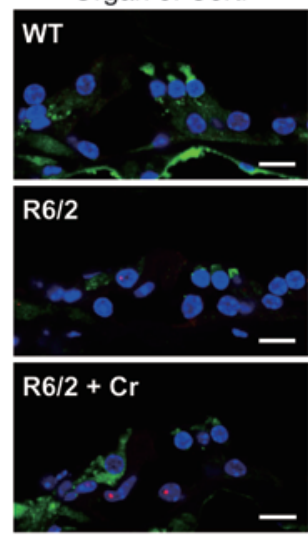

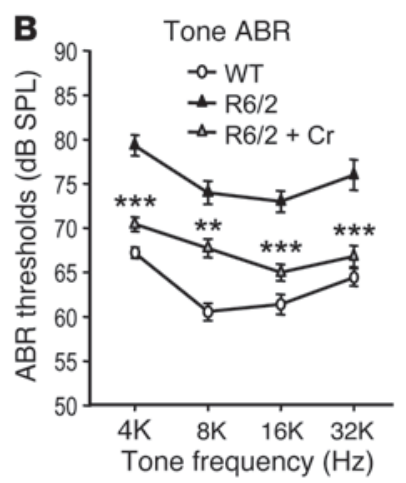
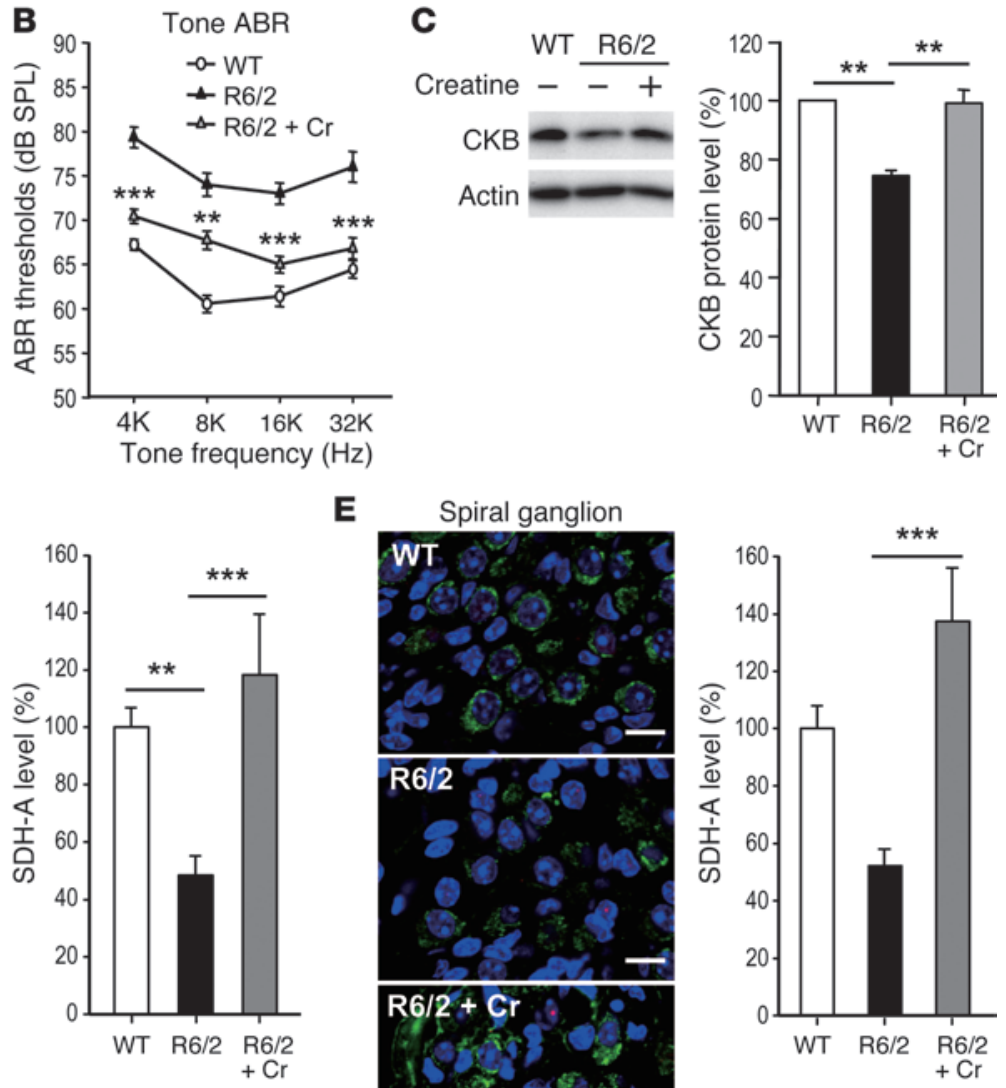

E

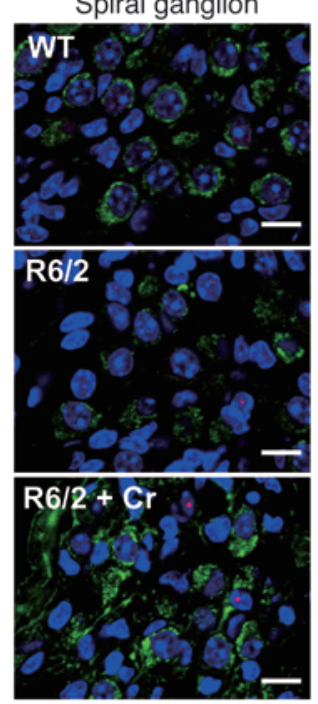

Figure 3

Beneficial effects of creatine supplementation on auditory dysfunction in HD mice. Male mice (WT, control diet, $n=18$; $\mathrm{R} 6 / 2$, control diet, $n=15$; and $\mathrm{R} 6 / 2$ mice, $2 \%$ creatine $[\mathrm{Cr}$, $n=11$ ) were fed the indicated diet from the age of 4 weeks. The ABR thresholds upon application of click stimuli (A) or tone-burst stimuli (B) to the indicated mice at the age of 10.5 weeks are shown. (C) Total lysates were harvested from cochlea at the age of 10.5 weeks. Expression of the CKB protein was determined using Western blot analyses and normalized to that of actin as an internal control. Quantitative data are shown in the right panel. Immunostaining of the organ of Corti (D) and spiral ganglion (E) of WT and $\mathrm{R} 6 / 2$ mice (10.5 weeks of age) was conducted to determine the expression of SDH-A (green) and NIls (red). Scale bars ( $\mathbf{D}$ and $\mathbf{E}$ ): $10 \mu \mathrm{m}$. Quantitative analyses are shown in the right panels. Data are presented as the mean \pm SEM. ${ }^{* \star} P<0.01$ and ${ }^{* * \star} P<0.001$.
The downregulation of CKB in the cochlea of HD mice is bound to weaken the creatine/PCr shuttle system catalyzed by $\mathrm{CKB}$ and might contribute to the hearing impairment of HD patients. To test this hypothesis, we supplemented the diet of R6/2 mice with creatine (the substrate of CKB; $2 \%$ ) for 7 weeks beginning at the age of 4 weeks. We found that creatine supplements ameliorated reduced body weight, motor coordination, and hearing deterioration in R6/2, but not WT, mice (Figure 3, A and B, and Supplemental Figure 4). Because dietary creatine supplement did not affect the number of NIIs (Supplemental Figure 6), formation of NIIs might not directly contribute to the hearing impairment observed in HD mice. Surprisingly, creatine supplementation also normalized the reduced expression of cochlear CKB (Figure 3C), which might further facilitate energy production in the cochlea. This observation indicates a potential positive feedback regulation between the $\mathrm{PCr}-\mathrm{CK}$ system and the expression of $\mathrm{CKB}$. Because creatine is an antioxidant (15) and chronic treatment with antioxidants is known to elevate the CKB level (16), dietary creatine supplement thus might rescue the expression of cochlear CKB in HD mice by reducing oxidative stress, thereby restoring their hearing. Moreover, creatine supplementation also normalized the downregulation of a major component of mitochondrial complex II (the succinate dehydrogenase Fp subunit SDH-A) in the cochlea of R6/2 mice (Figure 3, D and E). Reduction of SDH-A, a sign of mitochondrial dysfunction, has been reported in the brains of HD patients and mice $(17,18)$. By ameliorating mitochondrial deficit, creatine might further improve energy homeostasis. These observations strongly suggest that the CK system plays an important role in the cochlear dysfunction associated with HD. Most importantly, the hearing impairment in HD might be treatable by enhancement of the PCr-CK system. This is a timely issue, because a phase III trial of dietary creatine treatment for HD has been funded by the NIH (NCT00712426) and is currently recruiting HD patients to evaluate the therapeutic effect and safety of creatine for HD patients. Other therapeutic approaches to improve the hearing impairment of HD patients, including the enhancement of CKB expression in the cochlea by viral delivery, might also be worth evaluating in the future.

In summary, we demonstrated that HD patients and mice experience hearing impairment. Expression of mutant Htt in the cochlea and the concurrent loss of CKB might contribute to this auditory dysfunction. Most importantly, a dietary creatine supplement that enhanced the PCr-CK-dependent energy level improved hearing sensitivity in HD mice. To the best of our knowledge, this is the first report that describes hearing loss and its possible underlying mechanism in HD.

\section{Methods}

Detailed protocols are listed in Supplemental Methods.

Participants. HD patients were recruited from Chang Gung Memorial Hospital (CGMH) and Taipei Veterans General Hospital (TVGH) and agematched control subjects were recruited from Tri-Service General Hospital (TSGH) for a complete audiologic evaluation in the corresponding hospitals and TSGH. The protocol was approved by the Institutional Review 
Boards at Academia Sinica, CGMH, TVGH, and TSGH. Informed consent was obtained prior to any study-related procedures. A diagnosis of HD was established by a neurological examination and genetic assessment of CAG expansion in the HTT gene.

Hearing assessment in HD subjects. PTAs of all subjects were obtained at octave frequencies from 250 to $8,000 \mathrm{~Hz}$. The 4F-PTA of each ear was defined as the average of the PTTs at 500, 1,000, 2,000, and 4,000 Hz.

Animals and diet administration. Male R6/2 (B6CBA-Tg[HDexon1]62Gpb/1J) (12) and the knock-in HD mouse model ( $\left.\mathrm{Hdh}^{(\mathrm{CAG}) 150}, \mathrm{~B} 6.129 \mathrm{P} 2-\mathrm{Hdh}^{\text {tm2Detl }} / \mathrm{J}\right)$ (13) were originally purchased from The Jackson Laboratory. Animal experiments were performed under protocols approved by the Academia Sinica Institutional Animal Care and Utilization Committee.

ABRs of animals. ABRs were recorded as described previously (19) and detailed in Supplemental Methods.

Immunohistochemistry. Immunostaining of the mid-modiolar sections of decalcified cochlea embedded in paraffin was performed using the avidinbiotin-peroxidase complex (ABC) method as described previously (3). For immunofluorescence staining, cryosections $(6 \mu \mathrm{m})$ were stained with the desired antibody (3).

Western blot analysis. Equal amounts of protein were prepared for Western blot analyses as described previously (3).

RNA purification and real-time quantitative PCR. RNA collection and real-time quantitative RT-PCR (RT-qPCR) were conducted as described previously (3).

ATP determination in isolated hair cells. Hair cells isolated from the mouse organ of Corti at the age of 12 weeks, as described previously (20), were collected for ATP assay using an ATP Bioluminescence Assay Kit HS II (Roche).

Statistics. Data represent the mean \pm SEM, unless stated otherwise. The level of significance was set at $P \leq 0.05$. Detailed statistical analyses are described in Supplemental Methods.

\section{Acknowledgments}

This work was supported by grants from the Academia Sinica (AS-94TP-B17 and AS-97-TP-B02 to Y. Chern), the Institute of Biomedical Sciences/Academia Sinica (Clinical Research Center grant CRC98P03B to Y. Chern, C.M. Chen, and B.W. Soong), the National Science Council, Taiwan (NSC98-2314-B-016-017-MY3 to C.H. Wang), and Tri-Service General Hospital (TSGH-C99-40 to C.H. Wang).

Received for publication April 3, 2010, and accepted in revised form January 19, 2011.

Address correspondence to: Yijuang Chern, Institute of Biomedical Sciences, Academia Sinica, Nankang, Taipei 115, Taiwan. Phone: 886.2.26523913; Fax: 886.2.27829143; E-mail: bmychern@ ibms.sinica.edu.tw. Or to: Chih-Hung Wang, Department of Otolaryngology - Head and Neck Surgery, Tri-Service General Hospital, Taipei, Taiwan. Phone: 886.2.8792.3311, ext. 16363; Fax: 886.2.8792.3100; E-mail: chwang@ndmctsgh.edu.tw.
1. Martin JB, Gusella JF. Huntington's disease. Pathogenesis and management. NEngl J Med. 1986; 315(20):1267-1276.

2. [No authors listed]. A novel gene containing a trinucleotide repeat that is expanded and unstable on Huntington's disease chromosomes. The Huntington's Disease Collaborative Research Group. Cell. 1993;72(6):971-983.

3. Chiang MC, et al. The A2A adenosine receptor rescues the urea cycle deficiency of Huntington's disease by enhancing the activity of the ubiquitin-proteasome system. Hum Mol Genet. 2009; 18(16):2929-2942.

4. Beste C, Saft C, Gunturkun O, Falkenstein M Increased cognitive functioning in symptomatic Huntington's disease as revealed by behavioral and event-related potential indices of auditory sensory memory and attention. J Neurosci. 2008;28(45):11695-11702.

5. Saft C, Schuttke A, Beste C, Andrich J, Heindel W, Pfleiderer B. fMRI reveals altered auditory processing in manifest and premanifest Huntington's disease. Neuropsychologia. 2008;46(5):1279-1289.

6. Wallimann T, Wyss M, Brdiczka D, Nicolay K, Eppenberger HM. Intracellular compartmentation, structure and function of creatine kinase isoenzymes in tissues with high and fluctuating energy demands: the "phosphocreatine circuit" or cellular energy homeostasis. Biochem J. 1992;281(pt 1):21-40.

7. Shin JB, et al. Hair bundles are specialized for ATP delivery via creatine kinase. Neuron. 2007; 53(3):371-386.

8. Sorolla MA, Reverter-Branchat G, Tamarit J, Ferrer I, Ros J, Cabiscol E. Proteomic and oxidative stress analysis in human brain samples of Huntington disease. Free Radic Biol Med. 2008;45(5):667-678.

9. Bainbridge KE, Hoffman HJ, Cowie CC. Diabetes and hearing impairment in the United States: audiometric evidence from the National Health and Nutrition Examination Survey, 1999 to 2004 Ann Intern Med. 2008;149(1):1-10.

10. Clark JG. Uses and abuses of hearing loss classification. ASHA. 1981;23(7):493-500.

11. [No authors listed]. Unified Huntington's Disease Rating Scale: reliability and consistency. Huntington Study Group. Mov Disord. 1996;11(2):136-142.

12. Mangiarini L, et al. Exon 1 of the HD gene with an expanded CAG repeat is sufficient to cause a progressive neurological phenotype in transgenic mice. Cell. 1996;87(3):493-506.

13. Lin C-H, et al. Neurological abnormalities in a knock-in mouse model of Huntington's disease.
Hum Mol Genet. 2001;10(2):137-144.

14. Perluigi $M$, et al. Proteomic analysis of protein expression and oxidative modification in $\mathrm{r} 6 / 2$ transgenic mice: a model of Huntington disease. Mol Cell Proteomics. 2005;4(12):1849-1861.

15. Lawler JM, Barnes WS, Wu G, Song W, Demaree S. Direct antioxidant properties of creatine. Biochem Biophys Res Commun. 2002;290(1):47-52.

16. Opii WO, et al. Proteomic identification of brain proteins in the canine model of human aging following a long-term treatment with antioxidants and a program of behavioral enrichment: relevance to Alzheimer's disease. Neurobiol Aging. 2008;29(1):51-70.

17. Benchoua $A$, et al. Involvement of mitochondrial complex II defects in neuronal death produced by $\mathrm{N}$-terminus fragment of mutated huntingtin. Mol Biol Cell. 2006;17(4):1652-1663.

18. Browne SE, et al. Oxidative damage and metabolic dysfunction in Huntington's disease: selective vulnerability of the basal ganglia. Ann Neurol. 1997;41(5):646-653.

19. Wu WL, Wang CH, Huang EY, Chen CC. Asic3(-/-) female mice with hearing deficit affects social development of pups. PLoS ONE. 2009;4(8):e6508.

20. He DZ, Zheng J, Edge R, Dallos P. Isolation of cochlear inner hair cells. Hear Res. 2000;145(1-2):156-160. 